\title{
A Glance on Nail Involvement by Pemphigus Vulgaris
}

\section{Vitorino Modesto dos Santos*}

\section{Department of Internal Medicine, Armed Forces Hospital, Catholic University of Brasilia, Brasília-DF, Brazil}

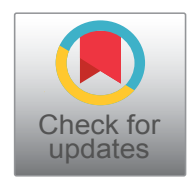

*Corresponding author: Dr. Vitorino Modesto dos Santos, Professor, Department of Internal Medicine, Armed Forces Hospital, Catholic University of Brasília, Estrada do Contorno do Bosque s/n, Cruzeiro Novo, CEP: 70630-900, Brasília-DF, Brazil, Tel: 55-61-32330812, Fax: 55-61-32331599, E-mail: vitorinomodesto@gmail.com

\begin{abstract}
Nail involvement by pemphigus vulgaris is uncommon and has been associated with the severity of disease. Paronychia, onychomadesis, Beau's lines, nail hemorrhages, cross riding, discoloration, hyperkeratosis, pitting, pterygium, onychodystrophy, onycholysis, onychorrhexis, onychoschizia and trachyonychia have been reported. These nail disorders may appear before, concomitantly or after the typical manifestations of pemphigus flare ups. Two adult patients presenting with nail disorders associated with pemphigus vulgaris are herein commented. Case studies might contribute to enhance the awareness about these nail disturbances very scarcely reported.
\end{abstract}

\section{Keywords}

Nail, Pemphigus vulgaris, Treatment

Pemphigus Vulgaris (PV) is an auto-immune entity involving the skin and mucosa, characterized by intraepithelial acantholytic bullous lesions [1-3]. Paronychia, onychomadesis and onycholysis are nail changes more often found in patients with PV, but these lesions are only rarely indicative of the bullous condition [1]. The suprabasal acantholysis and tombstone appearance are typical findings of biopsy study from the nail bed; and the direct and indirect immunofluorescence is needed to establish the final diagnosis of pemphigus [1-3]. The treatment of PV includes corticosteroid, azathioprine and dapsone, and nail changes can be adverse-effects.

I have read with interest the recent case study by Zawar, et al. describing recurrent paronychia, which appeared in all fingers and four toes of an Indian woman before her diagnosis of Pemphigus Vulgaris (PV) [2]. The 55-year-old patient presented hemorrhagic paronychia unresponsive to oral and topical antimicrobials long be- fore abrupt development of vesicular and bullous skin eruptions, and erosions in oral cavity, lips and eyes [2]. Correct diagnosis was based on clinical suspicion confirmed by typical histopathological findings, and direct and indirect immunofluorescence showing classical IgG and C3 deposition, and Dsg3 and Dsg1 titers of 1:40 [2]. She underwent oral prednisolone and azathioprine and had faster improvement of cutaneous than nail changes; paronychia recurred eight months later, associated with subungual hemorrhage and brittle nail on left index [2]. The authors emphasized the initial presentation of PV as chronic, further recurrent paronychia in multiple nails; additionally, they highlighted differences between the skin and mucosa and the bed, fold, and matrix of nails [2]. The relationship between the sites of lesions and respective morphological nail changes was commented too [2].

In this scenery, additional comments on a 32-yearold Brazilian man with PV might be of interest [3]. He presented with disseminated bullous and erosive cutaneous lesions, and oral, ocular and nasal erosions [3]. The diagnosis of PV was established by classical histopathologic features detected in the skin biopsy specimens, and prompt treatment with prednisone and dapsone was initially used, and further changed by azathioprine [3]. Worthy of note, in spite of successful improvement, multiple nail changes developed during convalescence [3]. These were characterized by onychomadesis, Beau's lines, transverse leukonychia, subungual hematoma, and onychodistrophy, thickening and transverse overcurvature of the nail plates as in initial stage of pincer nails [3]. Different from the Indian case, the nail changes appeared during convalescence, and

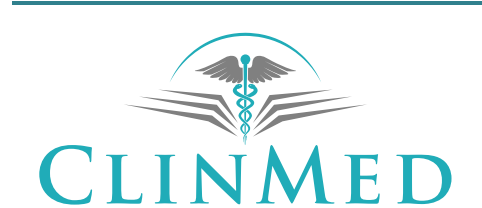

INTERNATIONAL LIBRARY

Citation: dos Santos VM (2017) A Glance on Nail Involvement by Pemphigus Vulgaris. Int J Pathol Clin Res 3:062. doi.org/10.23937/2469-5807/1510062

Received: September 18, 2017: Accepted: November 11, 2017: Published: November 13, 2017

Copyright: (c) 2017 dos Santos VM. This is an open-access article distributed under the terms of the Creative Commons Attribution License, which permits unrestricted use, distribution, and reproduction in any medium, provided the original author and source are credited. 
without paronychia $[2,3]$. The authors highlighted the early onset of successful treatment of the generalized lesions of PV and the late development of nail disorders; as well as their concern about an eventual drug-induced change in this setting [3].

Nail involvement by PV is considered uncommon and is often related to the severity of disease [1-3]. Although the nail lesions of PV can be easily observed by patients, their real origin may persist unsuspected. These changes will occur before, concomitantly or after the classical lesions of pemphigus, contributing to diagnostic challenges, under diagnosis or misdiagnosis, and late onset of adequate treatment of this disease [1-3].

In summary, early diagnoses can play a major role to reduce of the morbidity and mortality rates of PV; and authors believe that case reports would enhance the suspicion index of non-specialists about the associated nail disorders, which may follow misdiagnosed or underestimated by workers of primary health care attention.

\section{Financial Disclosure}

There was no grant support for this study.

\section{Disclosure of Potential Conflicts of Interest}

The authors had full freedom of manuscript preparation and there were no potential conflicts of interest.

\section{References}

1. Benhiba H, Hamada S, Guerouaz N, Saidi A, Senouci K, et al. (2013) Pemphigus vulgaris: An unusual clinical presentation. Ann Dermatol Vénéréol 140: 116-119.

2. Zawar V, Pawar M, Kumavat S (2017) Recurrent paronychia as a presenting manifestation of pemphigus vulgaris: A case report. Skin Appendage Disord 3: 28-31.

3. Santos VM, Azevedo MSA, Brito KRM, Luz LA, Ribeiro KD (2015) A young man with pemphigus and ungueal changes. Rev Med Saude Brasilia 4: 37-41. 